\title{
Análisis de las videotecas de las principales televisiones en España
}

\author{
Adrián De Mon Martín
}

\author{
Javier Guallar
}

Recibido: 14 de noviembre de 2014

Aceptado: 24 de noviembre de 2014

\section{Resumen}

El objetivo de este estudio es analizar los archivos audiovisuales de las principales televisiones de España mediante 24 indicadores agrupados en 5 apartados: aspectos generales, contenido, sistema de consulta, presentación de resultados y aspectos audiovisuales. La muestra analizada son las televisiones generalistas españolas con mayor audiencia durante 2013 (Antena 3, Cuatro, La Sexta, Telecinco y TVE1). Los resultados del estudio diferencian los mejores resultados del archivo público de TVE1 respecto al resto de televisiones privadas analizadas. En general, hay una tendencia a dar más importancia a la visualización del contenido que a la calidad de los sistemas de búsqueda.

\section{Pallabras clave}

Videotecas, Televisión, Audiencia, Documentación audiovisual, Documentación en televisión, Contenidos en línea, Investigación evaluativa.

\section{Analysis of the video archives of the main televisions in Spain}

\section{Abstract}

The aim of this study is to analyse the audiovisual archives on the Internet of the main televisions of Spain by 24 indicators, which are grouped into five sections: general aspects, content, query system, presentation of results and audiovisual aspects. The methodology follows the evaluation research with the technique of content analysis applied in other works to evaluate digital press archives or television archives. The analysed samples are the televisions with more audience in Spain during 2013 (Antena 3, Cuatro, La Sexta, Telecinco and TVE1). The results of the study difference the better results of the public archive of TVE1 with the rest of the private televisions analysed. In general, there is a tendency to give more importance to the display of content than to the quality of the search systems.

\section{Keywords}

TV archives, Television, Audience, Audiovisual documentation, TV documentation, Online content, Evaluative reesearch.

http://dx.doi.org/10.5209/rev CDMU.2014.v25.47472 


\section{INTRODUCCIÓN}

La televisión se encuentra a pocos años de cumplir el centenario desde su creación por John Logie Baird (será en 2024), y a pesar de sus diversos cambios a lo largo de este tiempo sigue siendo un medio de comunicación muy importante en nuestros días. Debido a este papel central de la televisión en la comunicación social a lo largo de su historia, los archivos de las empresas televisivas son una parte fundamental de la memoria audiovisual de los siglos XX y XXI.

En los últimos años, las televisiones se están volcando en su presencia en Internet, llevadas a ello por diversos factores, como la evolución de la tecnología que permite mejorar sustancialmente el almacenamiento y la transmisión de documentos audiovisuales a través de la red, o el creciente consumo de contenidos online por parte de las audiencias. Es así como "las cadenas de televisión han visto el potencial real de los archivos audiovisuales en línea y, en consecuencia, están aumentando el tipo de contenidos y las prestaciones de estos sistemas" (Antón y Guallar, 2014).

Ante esta situación, consideramos necesaria la existencia de estudios periódicos que desde la perspectiva de las ciencias de la información y la documentación, analicen la situación, características y prestaciones de los archivos de las televisiones en Internet, como es el objetivo del presente trabajo.

\section{Antecedentes}

Existen numerosos trabajos sobre documentación audiovisual en general, y, en concreto, sobre documentación en televisión, siendo éste por tanto un campo de estudio muy fértil en España tanto por parte de autores a nivel académico como profesional, si bien ya no son tan abundantes los trabajos sobre los archivos de las televisiones en la red.

Entre los autores y los trabajos que han estudiado la documentación televisiva en España se pueden citar, sin intención exhaustiva, a Caldera-Serrano en sus numerosos estudios, por ejemplo $(2013,2014)$ o junto a Arranz-Escacha (2012); López-de-Quintana (2007, 2014); Hidalgo Goyanes (2005, 2013); Agirreazaldegi Berriozabal (2007, 2011); Giménez Rayo (2007, 2013), López Yepes o Caridad et al (2012). En los textos citados se puede ver la evolución de las preocupaciones del sector en aspectos como la digitalización, la selección del material o las nuevas tendencias para los archivos y los profesionales. Asimismo, existen numerosos estudios de caso o descripciones de experiencias llevados a cabo por profesionales sobre el uso de la documentación en sus centros respectivos, de los que, debido a la gran variedad de ellos, excusamos no destacar ninguno en concreto.

Pero como señalábamos antes, sobre archivos audiovisuales o videotecas en línea, existen muy pocos trabajos, y a ellos nos vamos a referir ahora, así como a otros relacionados con metodología de investigación de archivos en la red que pueden ser de interés desde la perspectiva de nuestro estudio.

En cuanto a la metodología, son interesantes los estudios sobre evaluación de recursos en línea de Codina $(2000,2006)$ y los sucesivos análisis realizados para hemerotecas digitales por Guallar, Abadal y Codina (2009, 2012a, 2012b), con indicadores que permiten su adaptación a otros contextos, como son las televisiones. Ya entrando en el ámbito televisivo, otro tipo de metodología son las entrevistas (Agirreazaldegi, 2011) o las encuestas (Giménez-Rayo y Guallar, 2014) a responsables de centros de 
documentación de televisiones para conocer su dinámica de trabajo, si bien, nos interesan especialmente los estudios que tienen un planteamiento evaluativo, como los señalados antes para recursos web o hemerotecas, pero aplicados a fondos audiovisuales.

Aquí cabe señalar algunos trabajos: Rovira (2007) recoge datos de manera manual y automatizada sobre la calidad de los sitios web de televisiones locales catalanas, empleando una serie de indicadores, de los cuales dos tratan de sus archivos. Mendiguren (2010) estudia la presencia en Internet de un grupo de televisiones locales vascas combinando entrevistas personales con análisis de contenido de sus webs. Pérez Ribera (2012) analiza los servicios de acceso en línea al fondo audiovisual de las televisiones a través de una serie de indicadores, centrándose en el aspecto del servicio de venta y explotación de imágenes del archivo y sus derechos correspondientes, en una muestra de televisiones españolas y de países europeos. Caldera Serrano (2013) presenta una metodología para auditorías de colecciones audiovisuales, siendo especialmente de interés para este trabajo los apartados de análisis referentes a la interfaz de consulta y al contenido audiovisual. Por último, el trabajo de Antón y Guallar (2014), adapta el modelo de evaluación de hemerotecas de prensa al campo de los archivos audiovisuales añadiendo nuevos indicadores del campo audiovisual, y estudia las televisiones autonómicas españolas.

\section{OBJETIVOS Y METODOLOGÍA}

\section{Objetivos}

El objetivo principal de este trabajo es adaptar la metodología evaluativa de estudios anteriores al estudio de las televisiones generalistas de mayor audiencia en España, y se puede desglosar en estos objetivos específicos:

- Proponer un modelo evaluativo de indicadores aplicado a videotecas a partir de modelos anteriores.

- Evaluar las prestaciones y características de las videotecas de las televisiones generalistas de mayor audiencia en España.

\section{Metodología}

El trabajo sigue el método de investigación evaluativa (Abadal, 2006), donde la visión del problema a investigar se centra en juzgar la utilidad de unos sistemas de información concretos a través de unos criterios ya determinados, y la técnica de investigación aplicada ha sido el análisis de contenido de los archivos en línea de las televisiones de la muestra escogida.

La muestra de análisis son las cadenas de televisión españolas con mayor audiencia según el informe del Estudio General de Medios correspondiente a los meses de febrero a noviembre de 2013:

Antena 3: http://www.atresplayer.com/directos/television/antena3/

Cuatro: http://www.mitele.es/directo/cuatro/

La 1: http://www.rtve.es/noticias/directo-la-1/

La Sexta: http://www.atresplayer.com/directos/television/lasexta/

Telecinco: http://www.mitele.es/directo/telecinco/

Para analizar las características de los archivos audiovisuales en línea de las televisiones se han utilizado un total de 24 indicadores que se agupan en 5 apartados: aspectos generales, contenido, sistema de consulta, 
presentación de resultados y aspectos audiovisuales. Para establecer estos indicadores nos hemos basado en:

- Adaptar los establecidos para hemerotecas (Guallar y Abadal, 2009; Guallar, Abadal y Codina, 2012b) especialmente en los apartados de aspectos generales, sistema de consulta y presentación de resultados.

- Adaptar los indicadores del estudio sobre archivos en línea de televisiones autonómicas (Antón y Guallar, 2014), especialmente para el apartado de aspectos audiovisuales.

- Crear indicadores propios para características que se ha considerado que en otros estudios no han sido abarcadas suficientemente como la inserción de publicidad o la calidad del visionado en los apartados de contenido y aspectos audiovisuales.

Se ha seguido un sistema de puntuación propio aunque inspirado en los sistemas aplicados en estudios anteriores: 0 (indica un nivel bajo), 1 (nivel medio), 2 (nivel alto). Se ha querido homogeneizar la puntuación para todos los indicadores a estas tres posibilidades, de cara a poder tener mayores opciones comparativas entre todos los indicadores, respecto a otros estudios que cambian sus posibilidades según el indicador de 2 a 4 opciones de puntuar.

A partir de la muestra y de los indicadores mencionados, cuyo uso se detalla a continuación, el periodo de recogida de datos se ha realizado del 10 de abril al 10 de mayo de 2014.

\section{INDICADORES PARA LA EVALUACIÓN DE VIDEOTECAS}

Los indicadores propuestos son los siguientes:

APARTADO

\section{A. ASPECTOS GENERALES}

B. CONTENIDO
INDICADOR

A1. Denominación

A2. Ubicación

A3. Información del archivo

A4. Coste

B1. Tipología de programas

B2. Origen

B3. Cobertura temporal

B4. Actualización

B5. Anuncios publicitarios

C1. Tipos de consulta

C2. Lenguaje de interrogación

C3. Recuperación por navegación

C4. Opciones de filtro

C5. Ayuda

D1. Gestión de resultados

D2. Campos visualizados de cada registro

D3. Agrupación de resultados por tipología de programas 
E. ASPECTOS AUDIOVISUALES

D4. Identificación de términos de búsqueda

E1. Información básica

E2. Descripción del contenido

E3. Opciones de visionado

E4. Calidad del visionado

E5. Opciones de gestión del vídeo

E6. Enlaces relacionados

Tabla I. Indicadores a aplicar según su apartado correspondiente

A continuación se detalla el uso de cada indicador:

\section{A. ASPECTOS GENERALES}

INDICADOR DESCRIPCIÓN

A1. Denominación $\quad$ Términos utilizados por cada televisión para denominar a su archivo audiovisual en línea. Deben ser comprensibles y unívocos para que el usuario los identifique correctamente. No tiene puntuación al ser puramente informativo.

A2. Ubicación

Localización del acceso al archivo audiovisual en línea dentro de la página web de inicio de la televisión.

2. Ubicación en un acceso directo desde el menú principal.

1. Ubicación en algún menú secundario.

0 . Sin presencia en la página web de inicio.

A3. Información del Información general sobre las características generales del archivo archivo

audiovisual de la televisión.

2. Información exhaustiva sobre el archivo audiovisual.

1. Información básica.

0. No proporciona información.

A4. Coste

Evaluación de si hay coste por visualizar el contenido de los vídeos del archivo audiovisual.

2. Acceso gratuito.

1. Acceso parcialmente gratuito.

0 . Acceso totalmente de pago. 


\section{B. Contenido}

INDICADOR

B1. Tipología de programas

B2. Origen

B3. Cobertura temporal

B4. Actualización

B5. Anuncios publicitarios

\section{DESCRIPCIÓN}

Tipos de programas que se pueden encontrar: informativos, deportes, historia, entretenimiento, música, cultura, infantil.

2. Con 6 o 7 tipologías en el archivo audiovisual.

1. Con 4 a 5 tipologías.

0 . Menos de 4.

Procedencia de los contenidos mostrados en el archivo.

2. Material propio, coproducciones y ajeno.

1. Material propio y coproducciones.

0 . Material propio.

Alcance temporal de contenidos mostrados en el archivo.

2. Ofrece la totalidad temporal del archivo.

1. Ofrece más de 5 años de cobertura temporal del archivo.

0 . Ofrece menos de 5 años.

Tiempo que tarda en disponer del contenido en el archivo.

2. Disponible en línea el mismo día de emitirse.

1. Disponible en línea 1 día después de emitirse.

0 . Disponible en línea más de 1 día después de emitirse.

Inclusión de anuncios durante el visionado del contenido en línea.

2. Sin anuncios publicitarios durante todo el visionado.

1. Con anuncios publicitarios que se pueden parar durante el visionado.

0 . Con anuncios publicitarios que se han de visualizar.

\section{SISTEMA DE CONSULTA}

\begin{tabular}{|c|c|}
\hline INDICADOR & DESCRIPCIÓN \\
\hline C1. Tipos de consulta & $\begin{array}{l}\text { Opciones de búsqueda en el contenido del archivo en } \\
\text { línea. } \\
\text { 2. Búsqueda simple y avanzada. } \\
\text { 1. Búsqueda simple. } \\
\text { 0. Listado sin posibilidad de búsqueda. }\end{array}$ \\
\hline C2. Lenguaje de interrogación & $\begin{array}{l}\text { Búsqueda utilizando operadores booleanos (AND, OR, } \\
\text { NOT). } \\
\text { 2. Utiliza todos los operadores booleanos. } \\
\text { 1. Utiliza alguno pero no todos. }\end{array}$ \\
\hline
\end{tabular}




\section{0 . Sin operadores.}

C3. Recuperación por navegación Acceso a los documentos a través de índices: alfabético, temporal, por categorías o rankings (los más vistos, los más comentados).

2. Utiliza todos los tipos de índices y ranking.

1. Utiliza alguno pero no todos.

0 . No utiliza ningún tipo de índice ni de ranking.

C4. Opciones de filtro

Restricción de búsqueda por fecha de emisión o tipología de programa.

2. Utiliza todos los tipos de filtros.

1. Utiliza algún tipo de filtro.

0 . No utiliza ningún tipo de filtro.

C5. Ayuda

Texto informativo y explicativo sobre el funcionamiento del sistema de consulta y sus posibles búsquedas.

2. Con ayuda informativa y explicativa.

1. Con ayuda meramente informativa.

0 . Sin ayuda.

\section{PRESENTACIÓN DE RESULTADOS}

INDICADOR

D1. Gestión de resultados

D2. Campos visualizados de cada registro

\section{DESCRIPCIÓN}

Criterio de ordenación de los resultados (por relevancia o por fecha).

2. Más de un criterio a elegir en la ordenación de resultados.

1. Criterio preestablecido en la ordenación de resultados.

0 . Sin posibilidad de ordenación de resultados.

Número de campos mostrados para cada registro en la página de resultados.

2. Más de 5 campos en el registro de resultados.

1. De 3 a 5 campos en el registro de resultados.

0 . Menos de 3 campos en el registro de resultados.

Presentación de los resultados clasificados según su tipología de programas (informativos, deportes, actualidad, entretenimiento, música, cultura, infantil).

2. Agrupación de los resultados por tipología de programas. 
1. Agrupación de los resultados por otro criterio.

0 . Sin agrupación de los resultados.

D4. Identificación de términos de búsqueda
Mostrar de forma destacada los términos buscados para que el usuario pueda identificarlos en cada resultado.

2. Términos de búsqueda resaltados claramente.

1. Términos de búsqueda poco resaltados.

0 . No resaltados.

\section{E. ASPECTOS AUDIOVISUALES}

\section{INDICADOR}

E1. Información básica

\section{DESCRIPCIÓN}

Mostrar los elementos identificadores de cada documento audiovisual: título del programa, número y/o título del capítulo, tipología de programa, fecha de emisión, duración.

2. Tiene 5 o 6 identificadores del documento audiovisual.

1. Tiene de 2 a 4 identificadores.

0 . Sólo tiene 1 o ningún identificador.

\section{E2. Descripción del contenido}

Mostrar los elementos descriptivos del contenido
Posibilidades del sistema del archivo para visionar el contenido audiovisual: reproductores variados, pantalla completa, subtitulado.

2. Tiene las 3 opciones de visionado del contenido audiovisual.

1. Tiene 1 o 2 opciones.

0 . No tiene ninguna opción.

E4. Calidad del visionado

Opción de visionado de vídeo en varios formatos o en alta audiovisual: resumen, transcripción del audio y etiquetas.

2. Tiene los 3 descriptores del contenido audiovisual.

1. Tiene 1 o 2 descriptores.

0 . No tiene ningún descriptor. calidad (HD).

2. Tiene las 2 opciones de calidad en el visionado del vídeo.

1. Tiene 1 de las opciones.

0 . No tiene ninguna opción.

Posibilidades de poder gestionar cada vídeo: comentar, poner como favorito, votar, compartir en redes sociales y copiar URL.

2. Tiene las 5 opciones de gestión del vídeo.

1. De 2 a 4 opciones. 


\section{Tiene 1 o ninguna opción.}

E6. Enlaces relacionados

Posibilidad de, una vez visionado el vídeo, acceder a la web propia del programa/serie que muestra el vídeo o a otros vídeos relacionados del programa/serie.

2. Tiene las dos opciones de enlaces relacionados.

1. Tiene una.

0 . No tiene ninguna.

Tabla II. Indicadores definidos y con su puntuación a aplicar según su apartado correspondiente

\section{RESULTADOS}

\section{Resultados obtenidos}

Los resultados obtenidos según la puntuación de cada indicador para cada archivo televisivo en línea son los siguientes:

\begin{tabular}{|c|c|c|c|c|c|c|}
\hline INDICADOR & ANTENA 3 & TELE 5 & TVE 1 & LA SEXTA & CUATRO & TOTAL \\
\hline A1. Denominación & Atresplayer & Mitele & $\begin{array}{l}\text { RTVE a la } \\
\text { carta }\end{array}$ & Atresplayer & Mitele & - \\
\hline A2. Ubicación & 2 & 1 & 1 & 2 & 1 & $7 / 10$ \\
\hline $\begin{array}{l}\text { A3. Información del } \\
\text { archivo }\end{array}$ & 2 & 1 & 0 & 2 & 1 & $6 / 10$ \\
\hline A4. Coste & 1 & 2 & 2 & 1 & 2 & $8 / 10$ \\
\hline $\begin{array}{l}\text { B1. Tipología de } \\
\text { programas }\end{array}$ & 1 & 0 & 2 & 1 & 0 & $4 / 10$ \\
\hline B2. Origen & 1 & 2 & 1 & 2 & 2 & $8 / 10$ \\
\hline $\begin{array}{l}\text { B3. Cobertura } \\
\text { temporal }\end{array}$ & 1 & 1 & 2 & 1 & 1 & $6 / 10$ \\
\hline B4. Actualización & 2 & 2 & 2 & 2 & 2 & $10 / 10$ \\
\hline $\begin{array}{l}\text { B5. Anuncios } \\
\text { publicitarios }\end{array}$ & 0 & 0 & 2 & 0 & 0 & $2 / 10$ \\
\hline C1. Tipos de consulta & 0 & 1 & 2 & 0 & 1 & $3 / 10$ \\
\hline $\begin{array}{l}\text { C2. Lenguaje de } \\
\text { interrogación }\end{array}$ & 0 & 0 & 2 & 0 & 0 & $2 / 10$ \\
\hline $\begin{array}{l}\text { C3. Recuperación por } \\
\text { navegación }\end{array}$ & 1 & 1 & 2 & 1 & 1 & $6 / 10$ \\
\hline C4. Opciones de filtro & 0 & 0 & 2 & 0 & 0 & $2 / 10$ \\
\hline C5. Ayuda & 1 & 1 & 1 & 1 & 1 & $5 / 10$ \\
\hline $\begin{array}{l}D 1 . \quad \text { Gestión de } \\
\text { resultados }\end{array}$ & 0 & 0 & 2 & 0 & 0 & $2 / 10$ \\
\hline $\begin{array}{l}\text { D2. Campos } \\
\text { visualizados de cada } \\
\text { registro }\end{array}$ & 1 & 1 & 1 & 1 & 1 & $5 / 10$ \\
\hline
\end{tabular}




\begin{tabular}{|c|c|c|c|c|c|c|}
\hline $\begin{array}{lr}\text { D3. Agrupación } & \text { de } \\
\text { resultados } & \text { por } \\
\text { tipología } & \text { de } \\
\text { programas } & \end{array}$ & 0 & 0 & 0 & 0 & 0 & $0 / 10$ \\
\hline $\begin{array}{l}\text { D4. Identificación de } \\
\text { términos de búsqueda }\end{array}$ & 0 & 1 & 2 & 0 & 1 & $4 / 10$ \\
\hline E1. Información básica & 1 & 2 & 1 & 1 & 2 & $7 / 10$ \\
\hline $\begin{array}{l}\text { E2. Descripción del } \\
\text { contenido }\end{array}$ & 1 & 1 & 0 & 1 & 1 & $4 / 10$ \\
\hline $\begin{array}{l}\text { E3. Opciones de } \\
\text { visionado }\end{array}$ & 1 & 1 & 1 & 1 & 1 & $5 / 10$ \\
\hline $\begin{array}{l}\text { E4. Calidad del } \\
\text { visionado }\end{array}$ & 2 & 1 & 0 & 2 & 1 & $6 / 10$ \\
\hline $\begin{array}{l}\text { E5. Opciones de } \\
\text { gestión del vídeo }\end{array}$ & 1 & 1 & 1 & 1 & 1 & $5 / 10$ \\
\hline $\begin{array}{ll}\text { E6. } & \text { Enlaces } \\
\text { relacionados } & \end{array}$ & 2 & 1 & 2 & 2 & 1 & $8 / 10$ \\
\hline TOTAL & $21 / 46$ & $21 / 46$ & $31 / 46$ & $22 / 46$ & $21 / 46$ & \\
\hline
\end{tabular}

Tabla III. Resultados de las puntuaciones de cada indicador aplicadas a los archivos audiovisuales en línea analizados

\section{Análisis de resultados}

\section{A. Aspectos generales}

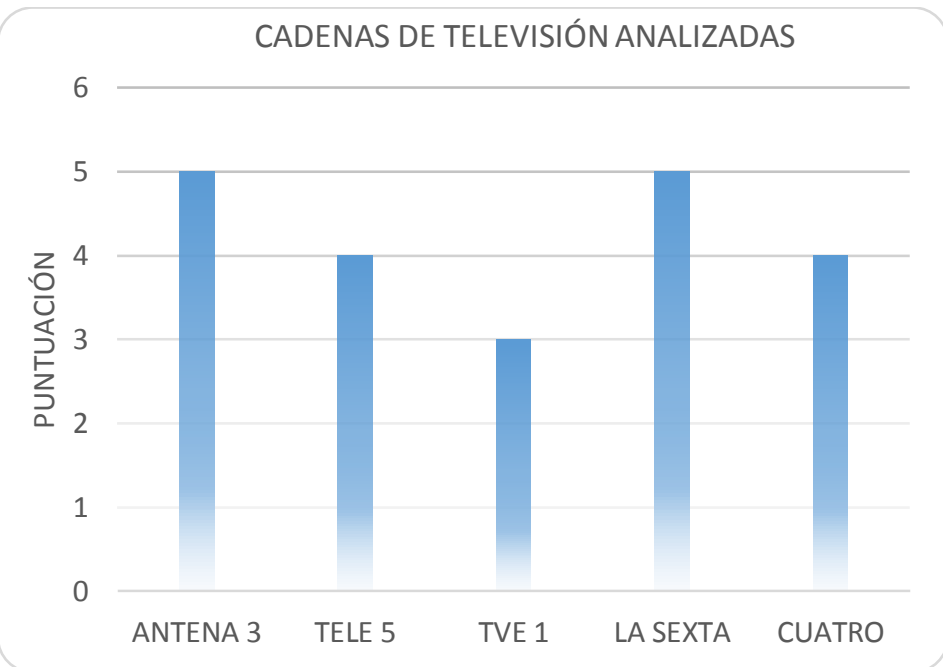

Gráfico 1. Puntuación obtenida sobre los aspectos generales de los archivos audiovisuales en línea

A1. Denominación: las televisiones generalistas de España presentan una divergencia a la hora de denominar a sus archivos audiovisuales. TVE es la única de la muestra estudiada que asigna el calificativo de "a la carta", denominando a su archivo corporativo de radio y televisión como "RTVE a la Carta". Por su parte 
las cadenas privadas analizadas, al haber entrado en procesos de fusión, tienen plataformas audiovisuales comunes, siendo "Atresplayer" la denominación del acceso a los vídeos de Antena 3 y La Sexta, y "Mitele" la de Tele 5 y de Cuatro.

A2. Ubicación: dentro de la página web inicial de las televisiones se muestran diversas prácticas de acceso a su archivo audiovisual. Mientras en las cadenas del grupo televisivo Atresmedia (Antena 3 y La Sexta) está destacado junto al menú principal y permite un rápido acceso, en Tele 5 y Cuatro se ofrece un menú secundario en forma de banner por encima de la web, lo que puede dificultar su visualización al parecer más un anuncio que un contenido propio del archivo. Por último, para acceder desde la web principal de RTVE se necesitan hasta 3 submenús hasta llegar al archivo concreto de TVE1, lo que dificulta la exploración al usuario.

A3. Información del archivo: este aspecto parece estar vinculado al de la ubicación del archivo al tener resultados similares según la importancia que se le ha dado a mostrar el archivo en un primer plano. Atresplayer explica de forma desglosada las posibilidades del archivo con las diferentes posibilidades según la tipología de usuario y a través de FAQ (Frequent Asked Questions), haciendo más fácil conocer cómo es su archivo. Por su parte, Telecinco y Cuatro tienen una información menos desarrollada y algo escondida en la subsección Consejos útiles, lo que puede dificultar encontrar a sus usuarios. Sorprende por último que en el archivo "TVE a la carta" no aparezca ninguna explicación al respecto.

A4. Coste: Sólo el archivo compartido de Antena 3 y La Sexta requiere pagar para acceder a ciertos contenidos a través de su sección Hazte Premium (que implica una suscripción mensual de 2,69 euros). Telecinco y Cuatro no exigen ningún pago en su archivo disponible; sólo en algunos casos registrarse de forma gratuita. Por su parte, el archivo de TVE 1 se puede visualizar sin pago ni registro alguno, en coherencia con su misión de servicio público.

\section{B. Contenido}

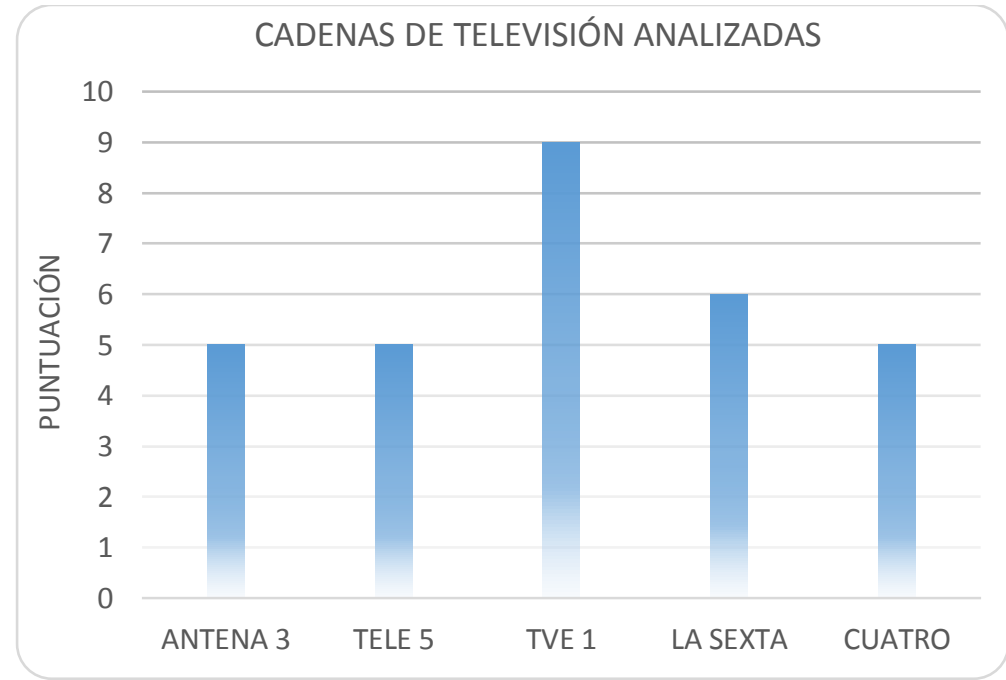

Gráfico 2. Puntuación obtenida sobre el contenido de los archivos audiovisuales en línea

B1. Tipología de programas: es un aspecto a considerar en los archivos audiovisuales para poder diferenciar sus programas y ofrecer una cierta diversidad. Así, las cadenas analizadas deberían mostrar ciertos tipos de programas como: informativos, deportes, historia, entretenimiento, música, cultura o infantil. TVE cumple con 6 de los 7 tipos, considerando las categorías de Archivo como historia y Magacín y series como entretenimiento, faltando los programas infantiles como categoría. Para Telecinco y Cuatro faltarían las secciones dedicadas a informativos, historia, música y cultura. Antena 3 y La Sexta no dan a conocer las categorías de informativos, historia y música. Por ello, vemos que se muestra una mayor 
diversidad de contenidos en la televisión pública, mientras que las televisiones privadas se basan más en el entretenimiento.

Categorías Ver todos los programas de la A a la Z

Informativos (227
Telediario
Los desayunos
Informe Semanal
Archivo (183)»
Fortunata y Jacint
La edad de oro
Un país en la moc
Cultura (237)»
Atención obras
Página Dos
Metrópolis

\section{Música (414)»}

Más que en vivo

Los conciertos de

Radio 3 en La 2

Los conciertos de La 2

\section{Series (137)》 \\ Isabel \\ Águila Roja \\ Cuéntame cómo pasó}

Documentales (263)»

La noche temática

Documentos TV

Documentales

culturales

Ciencia y
Tecnología (96)»
Aquí la Tierra
Zoom net
El escarabajo verde

\section{Deportes (138)» \\ Champions League \\ Baloncesto \\ Conexión tdp}

Magacín (154)»

La mañana

$T$ con $T$

Corazón

Figura 1. Tipología de programas (indicador B1) en el archivo en línea de RTVE

B2. Origen: la variedad del origen del material da riqueza al archivo ya sea a través del material propio, coproducido o ajeno. Tele 5 y Cuatro tienen disponibles online series ajenas a sus productoras como la serie Spartacus (emitida en Cuatro) o Intelligence (emitida en Telecinco). Antena 3 actualmente no tiene casi producciones ajenas pero puede emitirlas y La Sexta tiene algunas disponibles como El Mentalista de Estados Unidos. TVE1 no muestra producciones ajenas en su archivo. Todo ello muestra mayor peso de producciones de material ajeno en cadenas privadas con menos audiencia (Cuatro, La Sexta) que apuestan por un público especializado en series.

B3. Cobertura temporal: es un elemento muy importante para cualquier archivo, del tipo que sea. TVE ofrece un gran alcance temporal con buena parte de su archivo disponible (como programas de los años 50). Antena 3 muestra diversos programas o series de sus primeros años de emisión (como la serie Canguros de 1994). En La Sexta es difícil encontrar vídeos antiguos debido a su corta trayectoria, destacando un vídeo de 2006 del programa Sé lo que hicisteis. En Cuatro tienen una irregular cobertura temporal según los programas, teniendo en algunos todas sus temporadas como Cuarto Milenio (desde 2005). Telecinco muestra algunas series antiguas de su primera época como Al Salir de Clase (de 1997) pero no otras anteriores como Médico de Familia (de 1995). Con ello, vemos que sólo TVE ha hecho una apuesta profunda por digitalizar y poner en disposición la mayoría de su contenido audiovisual, el resto se han centrado en digitalizar algún contenido concreto. 


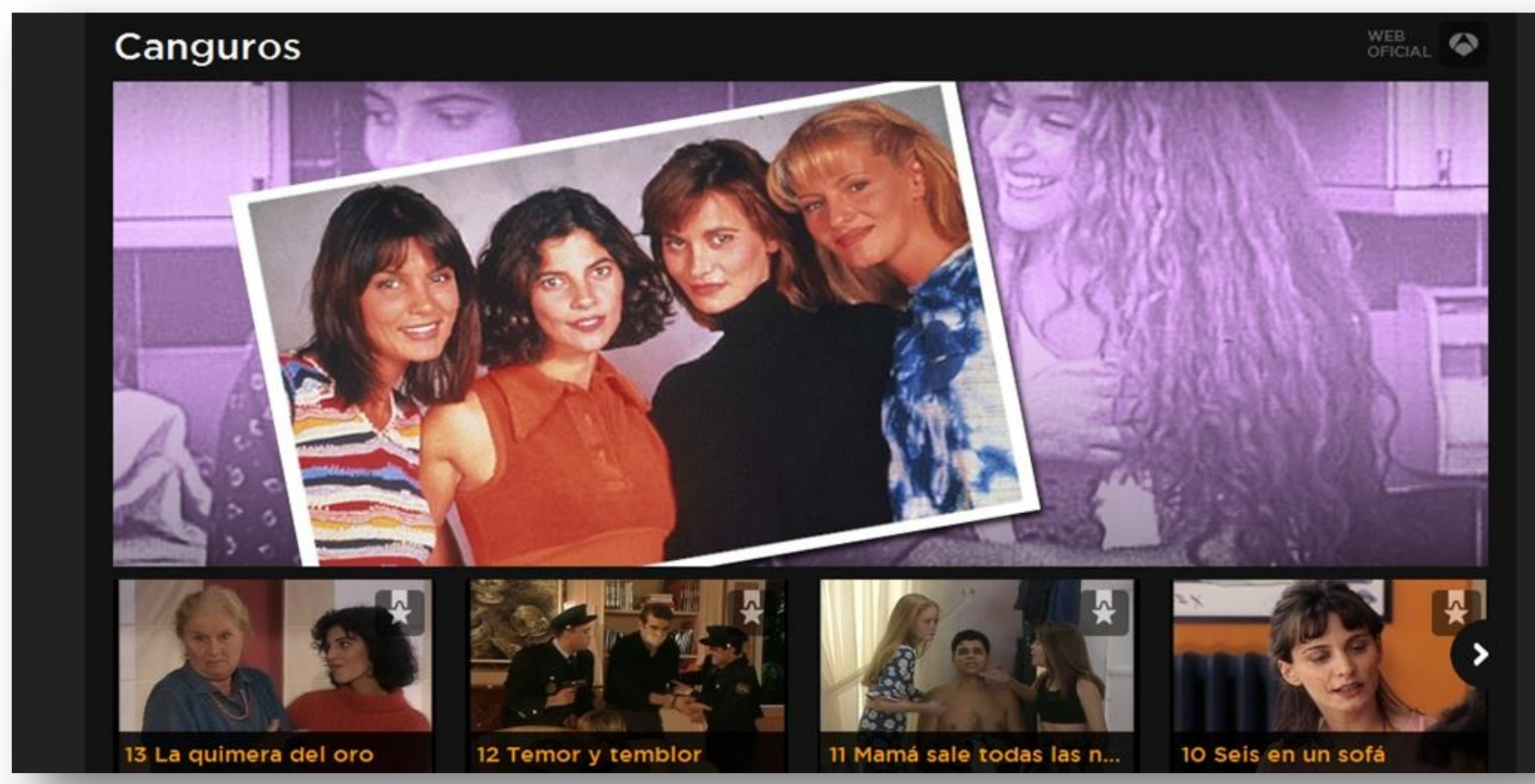

Figura 2. Serie de televisión Canguros de 1994 de Antena 3 como ejemplo de Cobertura temporal (indicador B3)

B4. Actualización: es uno de los factores clave para el éxito del archivo en línea, ya que los usuarios en Internet buscan la inmediatez para encontrar el contenido emitido. Todas las cadenas cumplen con su función inmediata: a los pocos minutos del final de la emisión, el contenido suele estar disponible en el archivo en línea además de haberlo podido ver en directo por Internet. Este indicador no da diferencias significativas entre las diferentes televisiones analizadas al ser para ellas un requisito básico.

B5. Anuncios publicitarios: Antena 3, La Sexta, Telecinco y Cuatro a través de sus respectivas plataformas de visualización insertan anuncios cada 10 o 20 minutos que se visualizan dentro del contenido del vídeo, los cuáles no se pueden parar ni saltar normalmente. TVE1 no muestra anuncios durante el visionado de sus programas debido a la política adoptada en los últimos años por el Gobierno de España de no financiarse con publicidad en la televisión pública. La diferente estrategia de financiación de las televisiones se ve claramente reflejada en este indicador, que puede llegar a ser molesto para el espectador. 


\section{Sistema de consulta}

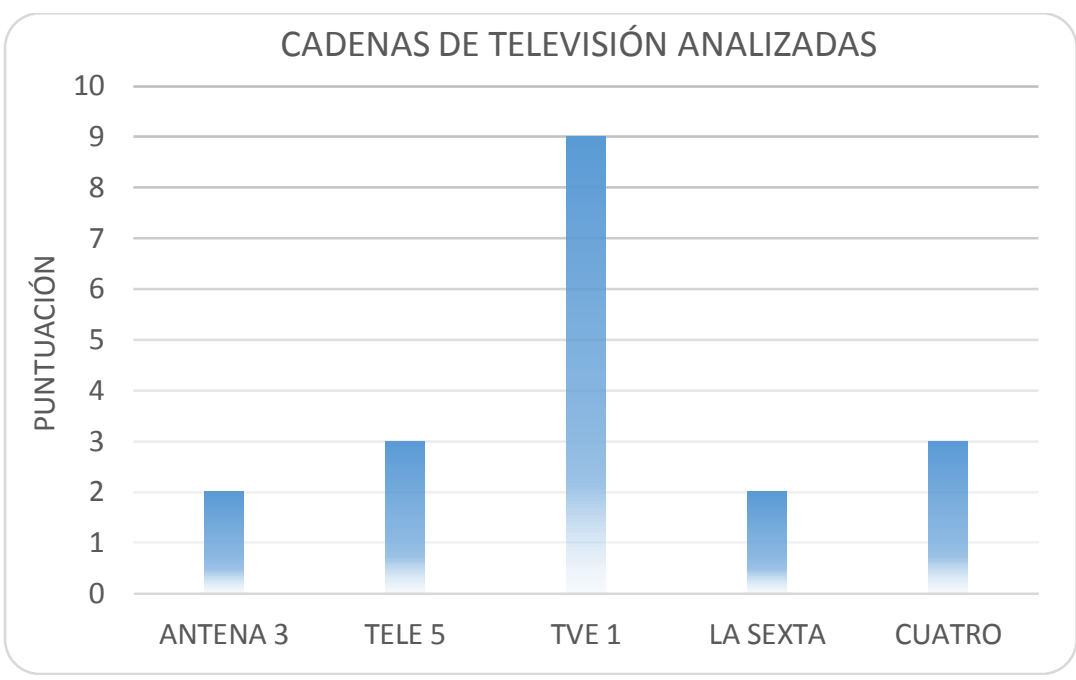

Gráfico 3. Puntuación obtenida sobre el sistema de consulta de los archivos audiovisuales en línea

C1. Tipos de consulta: las posibilidades de consulta al usuario son diversas entre la muestra estudiada. Antena 3 y La Sexta sólo permiten una búsqueda por listado alfabético del nombre de los programas. Telecinco y Cuatro ofrecen en su sistema una caja de búsqueda simple sin poder refinar resultados. Sólo el sistema de búsqueda de RTVE tiene búsqueda simple y avanzada (diferenciando esta última por tipo de contenido, sección, presencia, listado de resultados). Por tanto, de las televisisoes analizadas sólo el archivo en línea de RTVE pone a disposición de la audiencia en Internet un sistema de búsqueda eficiente.

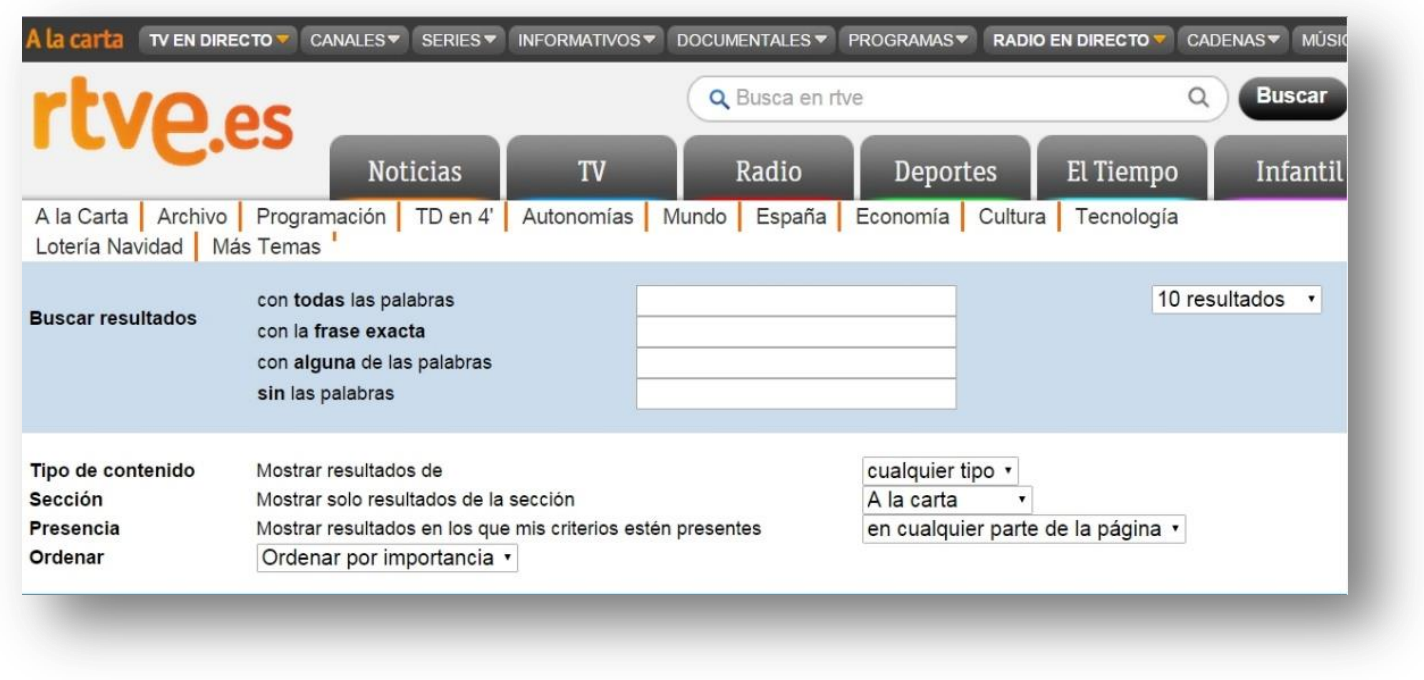

Figura 3. Búsqueda avanzada del archivo en línea de RTVE, ejemplo de Tipos de consulta (indicador C1) 


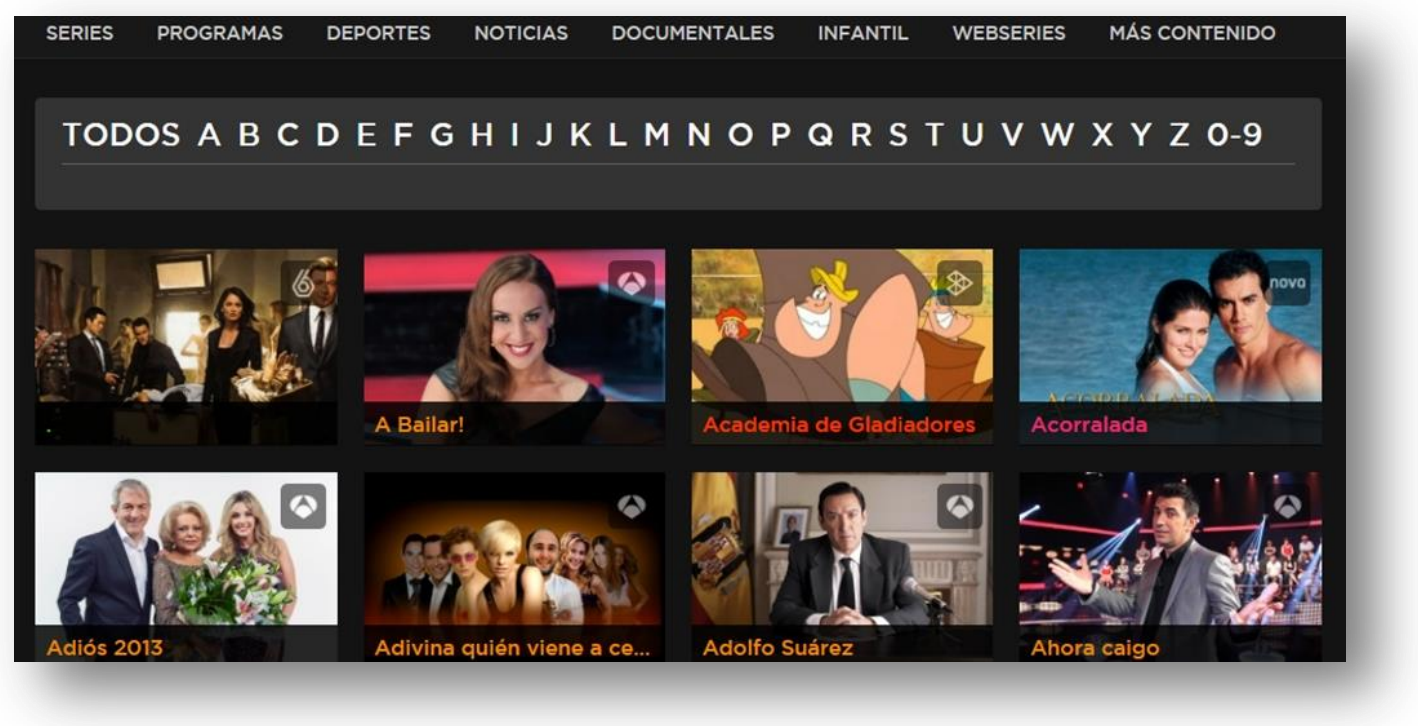

Figura 4. Búsqueda por listado alfabético de programas de Atresplayer (común para Antena 3 y La Sexta), ejemplo de Tipos de consulta (indicador C1)

C2. Lenguaje de interrogación: en relación con el indicador anterior, sólo el archivo en línea de RTVE tiene la posibilidad de utilizar una búsqueda con todos los operadores booleanos básicos, mientras que el resto de televisiones no ofrecen este tipo de lenguaje de interrogación. Todo ello acentúa la falta de desarrollo de los aspectos de recuperación de la información en las webs de las cadenas privadas de televisión.

C3. Recuperación por navegación: ninguna videoteca tiene todas las opciones de acceso a los documentos a través de índices: alfabético, temporal, por categorías o rankings (los más vistos, los más comentados). A TVE le falta la opción de rankings de los más vistos y de más comentados; aunque incorpore un porcentaje de popularidad a los vídeos, no permite recuperar por esta característica. Telecinco y Cuatro no ofrecen la cobertura temporal; y Antena 3 y La Sexta sólo tienen el índice alfabético sin mayores posibilidades de recuperación, mostrando por tanto una importante limitación.

C4. Opciones de filtro: la restricción de búsqueda por fecha de emisión o tipología del programa ha de ser un aspecto a valorar para refinar las búsquedas en el sistema. Así, RTVE ofrece los 2 tipos de filtros proporcionando muchas facilidades de encontrar resultados satisfactorios. El resto de televisiones no ofrecen estos filtros de recuperación. Aunque en Antena 3 y La Sexta se informa de la fecha de emisión de cada programa en el título, no es un campo indizado para ser recuperable por lo que el refinamiento de la búsqueda es nulo.

C5. Ayuda: las videotecas analizadas utilizan el sistema de preguntas frecuentes como única ayuda relacionada con el archivo audiovisual. Telecinco y Cuatro muestran una información muy básica para explicar la plataforma televisiva Mitele, con la posibilidad de contactar por mail si se necesita alguna ayuda concreta. Por su parte, Antena 3 y La Sexta se basan en numerosas preguntas frecuentes relativas a las diversas posibilidades de su plataforma Atresplayer relacionadas con la tecnología y según la tipología de sus usuarios (anónimo, registrado, suscriptor o premium). RTVE no tiene un apartado de ayuda específico sino un apartado llamado Contacto que responde a alguna mínima pregunta, con sólo una relacionada con el archivo en la sección RTVE.es. Por tanto, la ayuda de las videotecas debería ser más visible y completa para 
ser de utilidad a los usuarios de estas cadenas de televisión.

D. Presentación de resultados

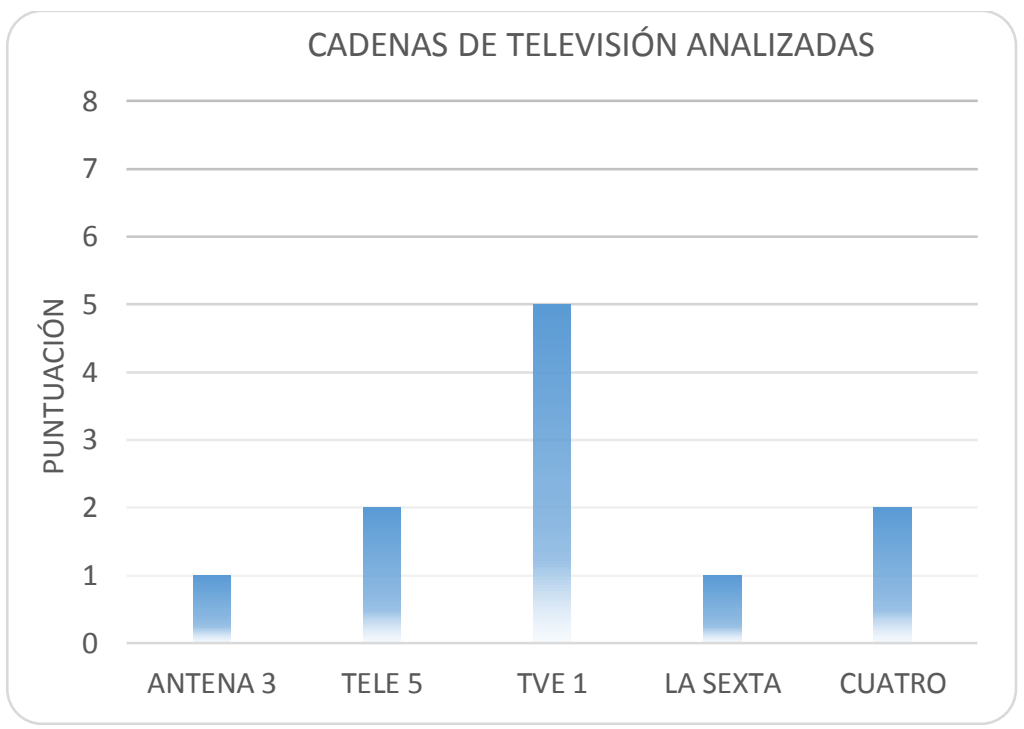

Gráfico 4. Puntuación obtenida sobre la presentación de resultados de los archivos audiovisuales en línea

D1. Gestión de resultados: el criterio de ordenación de los resultados obtenidos (por relevancia y por fecha) permitirá mayor rapidez de gestionar sus resultados. Telecinco y Cuatro no muestran ningún tipo de ordenación en su archivo, y La Sexta y Antena 3 no ofrecen una ordenación de los resultados al venir ya preestablecidos por orden alfabético. Por el contrario, TVE en su búsqueda avanzada sí que cumple los criterios de ordenación de resultados por relevancia (denominada "importancia") y por fecha.

D2. Campos visualizados de cada registro: según el número de campos mostrados para cada registro en la página de resultados se tendrá más facilidad para concretar un resultado. Ningún archivo muestra más de 5 campos en los registros de resultados, Antena 3 y La Sexta muestran 4 (título del programa, número de programa, sinopsis y fecha), Telecinco y Cuatro muestran además el género pero no la fecha en sus resultados, y, por su parte, RTVE no indica ni el género ni el número de programa. Por tanto, las videotecas deberían mostrar mayor información en sus resultados de búsqueda.

D3. Agrupación de resultados por tipología de programas: presentación de los resultados clasificados según su tipología de programas (informativos, deportes, actualidad, entretenimiento, música, cultura, infantil). Las videotecas analizadas no muestran agrupaciones por tipo de programas en sus resultados, sólo RTVE permite buscar dentro de sus secciones, pero no por sus tipos de programas.

D4. Identificación de términos de búsqueda: mostrar de forma destacada los términos buscados para que el usuario pueda identificarlos en cada resultado ha de ser una herramienta para identificar la pertinencia de los resultados. Sólo RTVE muestra los resultados resaltados donde aparecen esos términos, mientras Telecinco y Cuatro sólo indican los resultados de búsqueda generales a partir del término de búsqueda concreto sin resaltarlo en cada resultado que aparece. Antena 3 y La Sexta no tienen la posibilidad de búsqueda por término, sólo por listado alfabético. Son por tanto sistemas poco pensados para ofrecer al usuario un mejor refinamiento de búsqueda. 
E2. Descripción del contenido: mostrar los elementos descriptivos del contenido audiovisual: resumen, transcripción del audio y etiquetas. Ninguno cumple con los tres descriptores de contenido. Telecinco y Cuatro tienen resumen y etiquetas y sólo les falta la transcripción de audio. A Antena 3 y La Sexta les falta transcripción y etiquetas. Peor situación es la de RTVE con ningún descriptor de los requeridos, ya que el resumen es el del programa en general, no de cada programa específico y sin etiquetas sociales, sólo con breadcrumbs para poder volver a otros submenús de la web. En suma, estos elementos deberían mejorar para que antes de visionar un vídeo se pueda distinguir su contenido.

E3. Opciones de visionado: mostrar posibilidades amplias del sistema del archivo para visionar el contenido audiovisual como unos reproductores variados, opción de pantalla completa o el audio subtitulado. Ninguno de los archivos tiene las tres opciones de visionado, siendo las que tienen mayores prestaciones Telecinco y Cuatro donde sólo faltan los subtítulos y Antena 3 y La Sexta carecen de la opción de cambio de reproductor. RTVE sólo en ciertos vídeos ofrece la posibilidad de subtítulos y no tiene cambio de reproductor. Su implementación es necesaria para poder estar al nivel de los avances tecnológicos integrados en los diversos dispositivos de los usuarios que visualizan el archivo en línea, que requieren de actualizaciones constantes.

E4. Calidad del visionado: la opción de visionado en varios formatos o en alta calidad (HD) es un elemento extra a tener en cuenta para los vídeos visualizados por los usuarios. Antena 3 y La Sexta muestran hasta 6 opciones de calidad de vídeo para los usuarios registrados (Auto, SD, SD-, SD+, HD, HD+). Mientras, Telecinco y Cuatro, muestran pocas opciones de mejora en su visionado. RTVE no tiene ninguna opción de calidad de visionado. Sólo Atresplayer se ha interesado en este aspecto, por lo que el resto presentan limitaciones en la posibilidad de ofrecer mayor calidad de sus vídeos.

E5. Opciones de gestión del vídeo: ningún archivo tiene las cinco opciones de gestión, ya que no permiten copiar directamente la URL del vídeo pero sí la mayoría de opciones. En concreto, Antena 3 y la Sexta no permiten votar, Telecinco y Cuatro no permiten votar ni agregar como favorito y RTVE no deja comentar el vídeo concreto. Se trata de aspectos bastante desarrollados debido a la importancia de difundir el contenido de los vídeos y del archivo en línea entre los usuarios en las redes sociales.

E6. Enlaces relacionados: se trata de, una vez visionado el vídeo, poder acceder a la web del programa o de la serie. RTVE, Antena 3 y La Sexta tienen las 2 opciones de enlaces relacionados con enlaces a las webs específicas del programa o serie dentro de la web corporativa de cada televisión, mientras que Telecinco y Cuatro no lo muestran en su archivo. Los videos relacionados de otros programas o capítulos están en todos los archivos, aunque solo en RTVE se muestra al completo.

\section{CONCLUSIONES}

Los resultados obtenidos, aplicados a cada una de las televisiones nos permiten señalar lo siguiente:

TVE1 utiliza la plataforma corporativa de RTVE y lidera con la puntuación más alta en global de todas las televisiones analizadas. Destaca el coste gratuito de todo su contenido sin necesidad de registro, la variedad de tipologías de programas asignadas que favorecen otros indicadores como las opciones de filtro (por programa y por fecha), la gestión de ordenación de resultados según la importancia o fecha, donde el resto no ofrecen ninguna opción, su cobertura temporal y la búsqueda avanzada con uso operadores booleanos. Como aspectos negativos se puede señalar su mala agrupación de resultados disponibles y la inexistente opción de cambiar la calidad de visionado de los vídeos. 
Antena 3 y La Sexta se basan en la plataforma audiovisual Atresplayer obteniendo una puntuación mediabaja. Destacan de forma positiva su ubicación e información del archivo audiovisual dentro de la web corporativa, la calidad del visionado con importantes posibilidades al usuario para elegir su formato ideal y la presencia de los enlaces relacionados. Lo más negativo es todo lo referente a su sistema de consulta, que se basa simplemente en un listado alfabético, y sin permitir la búsqueda por palabra clave o interrogación. A destacar también en negativo la molesta inserción de anuncios cada 10 o 15 minutos de visionado de un vídeo.

Telecinco y Cuatro se muestran a través de la plataforma audiovisual Mitele y alcanzan una puntuación media-baja, siendo prácticamente similares a sus respectivos rivales de audiencias televisivas de Atresmedia. Los aspectos positivos para los usuarios son la no necesidad de registro y el mostrar cierta información básica del archivo audiovisual. Pero también resaltan negativamente las pocas tipologías de programas con una limitación en la agrupación de resultados y en sus opciones de filtro, así como la limitación del cajetín de búsqueda simple sin ningún lenguaje de interrogación. También muestra anuncios cada 20 minutos sin poderlos parar o saltarlos.

Un aspecto reseñable es la similar puntuación obtenida por las cuatro cadenas privadas analizadas, con puntuaciones casi idénticas a pesar de mostrar diferencias en cada indicador, quedando patente su poca atención hacia los aspectos más relacionados con la recuperación de la información y la gestión de resultados, y evidenciando una estrategia hacia los usuarios consistente en enseñar los contenidos del archivo de la forma más sencilla posible sin opciones de refinamiento y con una consulta rápida por listado o por búsqueda simple.

Por su parte, la televisión pública estatal española sí que ofrece a la audiencia mayor cobertura temporal y prestaciones, evidenciando que su videoteca está pensada no sólo para una búsqueda rápida sino también para que cualquier persona pueda encontrar información audiovisual en un amplio rango temporal.

El análisis global de los archivos de las principales televisiones generalistas en España permite señalar que su cada vez mayor cobertura temporal y la variedad de los contenidos que ofrecen contrasta con unos sistemas de consulta que están claramente poco desarrollados, conclusión similar a la del estudio sobre televisiones autonómicas de Anton y Guallar (2014).

Los nuevos hábitos de consumo de medios, que muestran a una audiencia cada vez más interesada por la televisión en Internet, deberían llevar a las empresas televisivas a potenciar el acceso y especialmente el sistema de consulta de sus archivos en línea, algo en lo que, como se ha señalado, existe un amplio margen de mejora.

Una posible continuación del trabajo aquí presentado es la de complementar la metodología evaluativa con entrevistas o encuestas a responsables de archivos audiovisuales. Por otra parte, además de proseguir con estudios evaluativos periódicos de las videotecas en línea, sería interesante comparar su situación con la de las hemerotecas y las audiotecas en línea, especialmente de estas últimas, poco tratadas en trabajos académicos. Se puede decir que la comparación de prestaciones ente hemerotecas y videotecas en base a los estudios existentes sitúa aquellas a un nivel más avanzado que éstas, pero sin duda son necesarias más 
investigaciones para completar una visión de conjunto periódicamente actualizada de los archivos de los medios de comunicación en Internet.

\section{REFERENCIAS BIBLIOGRÁFICAS}

Abadal, Ernest (2006). Mètodes i tècniques de recerca en Biblioteconomia i Documentació: fases de la recerca. http://eprints.rclis.org/15722/2/2-\%20Fasesalumnes.pdf (2014-10-10).

Agirreazaldegi Berriozabal, Teresa (2007). Claves y retos de la documentación digital en televisión. // El profesional de la información. 16:5 (septiembre-octubre 2007), 433-442. http://www.elprofesionaldelainformacion.com/contenidos/2007/septiembre/05.pdf. (2014-10-10).

Agirreazaldegi Berriozabal, Teresa (2011). La gestió de materials audiovisuals de programes informatius a les cadenes de televisió generalistes. // BiD: Textos Universitaris de Biblioteconomia i Documentació. 26 (junio 2011). http://www.ub.edu/bid/26/agirreazaldegi1.htm (2014-10-10).

Antón, Laura; Guallar, Javier (2014). Análisis de los archivos audiovisuales en Internet de las televisiones autonómicas españolas. // Revista española de documentación científica. 37:1 (enero 2014). http://redc.revistas.csic.es/index.php/redc/article/viewArticle/836/1071. (2014-10-10).

Caldera-Serrano, Jorge (2013). Metodología para el análisis de repositorio institucional de colecciones audiovisuales digitales. // Documentación de las Ciencias de la Información. 36 (2013) 209-219. http://dx.doi.org/10.5209/rev_DCIN.2013.v36.42109 (2014-10-10).

Caldera-Serrano, Jorge (2014). Realidad aumentada en televisión y propuesta de aplicación en los sistemas de gestión documental. // El profesional de la información. 23:6 (noviembre-diciembre 2014), 643-650. http://dx.doi.org/10.3145/epi.2014.nov.12 (2014-11-10).

Caldera-Serrano, Jorge; Arranz-Escacha, Pilar (2012). Documentación audiovisual en televisión. Colección El profesional de la información; 13. Barcelona: Editorial UOC, 2012.

Caridad Sebastián, Mercedes [et al.] (2011). Documentación audiovisual: nuevas tendencias en el entorno digital. Colección Comunicación audiovisual; 11. Madrid: Síntesis, 2011.

Codina, Lluís (2000). Evaluación de recursos digitales en línea: conceptos, indicadores y métodos. // Revista $\begin{array}{llllll}\text { Española de } \quad \text { Documentación } & \text { Científica. 23:1 } & \text { (enero-marzo } & 2000 \text { ) } & \text { 9-44. }\end{array}$ http://redc.revistas.csic.es/index.php/redc/article/view/315/479 (2014-10-10).

Codina, Lluís (2006). Metodología de análisis y evaluación de recursos digitales en línea. Barcelona: UPF, Área de Biblioteconomía y Documentación, Departamento de Periodismo y Comunicación Audiovisual. http://www.lluiscodina.com/metodos/metodos2006.doc (2014-10-10)

Giménez-Rayo, Mabel (2007). Documentación audiovisual de televisión: la selección del material. Colección Biblioteconomía y administración cultural; 171. Gijón: Trea, 2007. 
Giménez-Rayo, Mabel (2012). La documentación audiovisual en televisión en el mundo 2.0: retos y oportunidades. $\begin{array}{lllll}\text { // } & \text { Trípodos. } & 31 & \text { 79-97. }\end{array}$ http://tripodos.com/index.php/Facultat Comunicacio Blanquerna/article/download/39/24 (2014-10-10)

Giménez-Rayo, Mabel; Guallar, Javier (2014). Centros de documentación en televisión y productos documentales. // El profesional de la información. 23:1 (enero-febrero 2014) 13-25. http://dx.doi.org/10.3145/epi.2014.ene.02 (2014-10-10).

Guallar, Javier; Abadal, Ernest (2009). Evaluación de hemerotecas de prensa digital: indicadores y ejemplos de buenas prácticas. // El profesional de la información. 18:3(mayo-junio 2009) 255-269.

http://www.elprofesionaldelainformacion.com/contenidos/2009/mayo/02.pdf. (2014-10-10).

Guallar, Javier; Abadal, Ernest; Codina, Lluís (2012a). Hemerotecas de prensa digital. Evolución y tendencias. // El profesional de la información. 21:6 (noviembre-diciembre 2012) 595-605. http://hdl.handle.net/10760/18199 (2014-10-10).

Guallar, Javier; Abadal, Ernest; Codina, Lluís (2012b). Sistema de análisis de hemerotecas de prensa digital. // Trípodos. 31 (2012) 37-64.

http://www.tripodos.com/index.php/Facultat_Comunicacio_Blanquerna/article/view/37/22 (2014-10-10).

Hidalgo Goyanes, Paloma (2005). La documentación audiovisual de las televisiones. La problemática actual y el reto de la digitalización. // Documentación de las ciencias de la información. 28 (2005) 159-171.

http://revistas.ucm.es/index.php/DCIN/article/viewFile/DCIN0505110159A/19216. (2014-10-10).

Hidalgo Goyanes, Paloma (2013). Patrimonio audiovisual en televisión. // Marcos Recio, Juan Carlos (coords.). Gestión del patrimonio audiovisual en medios de comunicación. Colección Ciencias de la información. Comunicación audiovisual; 18. Madrid: Síntesis, 2013. 53-82.

López-de-Quintana, Eugenio (2007). Transición y tendencias de la documentación en televisión: digitalización y nuevo mercado audiovisual. // El profesional de la información.16:5 (julio-agosto 2007) 397-408. http://www.elprofesionaldelainformacion.com/contenidos/2007/septiembre/01.pdf. (2014-10-10).

López-de-Quintana, Eugenio (2014). Rasgos y trayectorias de la documentación audiovisual: logros, retos y quimeras. // El profesional de la información. 23:1 (enero-febrero 2014) 5-12. http://elprofesionaldelainformacion.com/contenidos/2014/enero/01.pdf (2014-10-10).

Mendiguren Galdospin, Teresa (2010). Análisis comparativo de las páginas web de las televisiones locales de la comarca del Gran Bilbao. // Zer: revista de estudios de comunicación . 15:29 (2010), 89-114. http://www.ehu.es/zer/hemeroteca/pdfs/zer29-05-Mendiguren.pdf (2014-10-10).

Pérez Ribera, Sonia (2012). Anàlisi dels serveis d'accés online al fons audiovisual de les televisions Directora: Luisa Tolosa Robledo. Projecte Fi de Carrera de la Llicenciatura en Documentació. Universitat Politècnica de València. http://hdl.handle.net/10251/16598 (2014-10-10).

Rovira Fontanals, Cristòfol (2007). Anàlisi i avaluació dels llocs web de televisions en català. // BiD: Textos Universitaris de Biblioteconomia i Documentació. 18 (Junio 2007 ). http://www2.ub.edu/bid/consulta_articulos.php?fichero=18rovir3.htm (2014-10-10). 